\title{
A class of systems with measurable Hannay angles
}

\author{
Simon Golin†£ and Stefano Marmi $\Varangle$ \\ † Fachbereich Mathematik, Technische Universität Berlin, Str. des 17. Juni 136, \\ D-1000 Berlin 12, Federal Republic of Germany \\ ‡ Dipartimento di Fisica, Università di Bologna, Via Irnerio 46, I-40126 Bologna, \\ Italy
}

Received 17 July 1989, in final form 2 November 1989

Accepted by M V Berry

\begin{abstract}
The Hannay angles arise in classical mechanics as an anholonomy effect for adiabatically time-dependent Hamiltonian systems. It is proven that for a class of systems with several degrees of freedom the Hannay angles can be experimentally investigated. Our method consists in averaging over the torus of initial angles of the motion.
\end{abstract}

AMS Classification scheme numbers: $34 \mathrm{C} 29,70 \mathrm{H} 99$

PACS numbers: 0320

\section{Introduction}

In 1985, Hannay [19] discovered an anholonomy effect in classical mechanics closely corresponding to the Berry phase $[7,8]$ in quantum mechanics and subsequently the term Hannay angles was coined. Until recently a difficulty in the experimental investigation of the Hannay angles seemed to have escaped attention. This was pointed out in [14], where averaging over the initial torus was suggested as a solution to the problem.

Our objective here is to present a class of systems with several degrees of freedom that allow a measurement of the Hannay angles. We announced our results in [17].

More specifically, we consider a class of adiabatically time-dependent Hamiltonian systems whose Hamiltonian is of the form

$$
h=h_{0}(J)+\varepsilon h_{1}(J, \varphi, \varepsilon t)
$$

where $(J, \varphi) \in \mathbb{R}^{n} \times \mathbb{T}^{n}$ are action-angle variables ( $\mathbb{T}^{n}$ being the $n$-dimensional torus). For small $\varepsilon>0$ the quantity $\tau:=\varepsilon t$ is slow time. We assume $h_{1}$ to be $T$-periodic in $\tau$ for some positive $T$. The crucial hypothesis under which the theorems of this paper will be formulated is that $h_{0}$ be $\tau$-independent, as opposed to the more general case otherwise considered in the context of Hannay angles. We point out that (1) is a special case of Hamiltonians describing slowly time-dependent

$\S$ Present address: Wissenschaftszentrum Nordhein-Westfalen, Reichsstrasse 45, D-4000 Düsseldorf 1 , Federal Republic of Germany. 
systems that are integrable in their instantaneously frozen frame. However, the class of systems described by (1) is rather wide. In particular, integrable timeindependent systems that are slowly rotated (in coordinate space) give rise to a Hamiltonian of the form $h=h_{0}(J)+\varepsilon h_{1}(J, \varphi, \tau)+\varepsilon^{2} h_{2}(J, \varphi, \tau)$, where $\varepsilon h_{1}$ contains the generalised potential of the Coriolis force and $\varepsilon^{2} h_{2}$ contains the potential of the centrifugal force and of the additional term arising if the angular velocity is not constant. Since in what follows we will only be interested in times $t \in[0, T / \varepsilon]$, the contributions to evolution coming from $\varepsilon^{2} h_{2}$ may be neglected (as on this time scale they give errors of order $\mathcal{O}(\varepsilon)$ ). We also remark that the only two examples of systems with more than one degree of freedom and non-trivial Hannay angles known to us, the Foucault pendulum and a satellite moving around a slowly rotating oblate or prolate planet $([20,21,16])$, belong to this class.

Set $f(J, \varphi, \tau):=-\nabla_{\varphi} h_{1}(J, \varphi, \tau), \quad g(J, \varphi, \tau):=\nabla_{J} h_{1}(J, \varphi, \tau), \quad$ and $\quad \omega(J):=$ $\nabla_{J} h_{0}(J)$; the Hamiltonian equations of motion are

$$
j=\varepsilon f(J, \varphi, \tau) \quad \dot{\varphi}=\omega(J)+\varepsilon g(J, \varphi, \tau) .
$$

Here the overdot denotes the derivative with respect to time $t$. The adiabatic Hannay angles are defined for small $\varepsilon>0$ by

$$
\begin{aligned}
\Delta \varphi & :=\varphi\left(\frac{T}{\varepsilon}\right)-\varphi^{0}-\int_{0}^{T / \varepsilon} \omega(J(t)) \mathrm{d} t \\
& =\varepsilon \int_{0}^{T / \varepsilon} g(J(t), \varphi(t), \varepsilon t) \mathrm{d} t
\end{aligned}
$$

where $\varphi^{0}:=\varphi(0)$.

Averaging theory will allow us (cf proposition 1 in section 2 and also $[13,16]$ ) to replace the adiabatic Hannay angles by the geometrical Hannay angles

$$
\int_{0}^{T} g^{\mathrm{av}}\left(J^{0}, \tau\right) \mathrm{d} \tau
$$

where $J^{0}:=J(0)$ and $g^{\text {av }}(J, \tau):=\int_{0}^{2 \pi} \ldots \int_{0}^{2 \pi} g(J, \varphi, \tau) \mathrm{d}^{n} \varphi /(2 \pi)^{n}$. The geometrical origin of (4) is discussed in $[16,15,18,24,27]$. What makes the averaged quantity (4) useful is that it can be determined without solving the non-autonomous system (2).

In [14] it was argued that in order to measure the Hannay angles experimentally one has to measure $\varphi(T / \varepsilon)$ and to complement this by an a priori estimate on the dynamical angles $\int_{0}^{T / \varepsilon} \omega(J(t)) \mathrm{d} t$. According to (3), this then allows the determination of the Hannay angles and the comparison of theory and experiment.

Unfortunately, there is no easy way of estimating the dynamical angles: neither can one solve (2) and determine $J(t)$ explicitly nor can one directly invoke averaging theory. In fact, one of our main incentives in [16] was to apply averaging theory to the geometrical Hannay angles. As we will show, if the Hamiltonian is of the form (1), then the estimates of [16] can be improved and averaging theory will allow us to prove under certain conditions that $\max _{t \in[0, T / \varepsilon]}\left|J(t)-J^{0}\right|=\mathcal{O}\left(\varepsilon^{1-2 a}\right)$ (as $\varepsilon \rightarrow 0$ for suitable $a \in\left[0, \frac{1}{2}\right.$ ) and for large sets of initial values (cf remark 1 after the proof of the proposition in section 2)). Nevertheless the naive approximation $T \omega\left(J^{0}\right) / \varepsilon$ to the dynamical angles is too crude: the error is of order $\mathcal{O}\left(\varepsilon^{-a}\right)$, i.e. it exceeds the Hannay angles (which are of order $\mathcal{O}(1)$ ).

We will prove that for multi-frequency systems with Hamiltonians of the form 
(1) the dynamical angles averaged over the initial angles $\varphi^{0}$ can be replaced (up to a small error term) by the simpler quantity $T \omega\left(J^{0}\right) / \varepsilon$. Consequently, the systems under consideration in this paper admit, in principle, for measurements of the Hannay angles (cf the discussion in section 3).

We emphasise that the arguments used for proofs are standard, and consequently they will only be sketched. In fact, our motivation is to present an argument related to the experimental verification of the Hannay angles and not to provide new techniques for dealing with adiabatic invariance. The remarks following the proposition briefly summarise the improvements which are possible for the class of Hamiltonians (1) with respect to the general situation (cf, e.g., [3, 16]).

We now summarise the paper. In section 2 we prove an adiabatic theorem and a statement concerning the averaging of phase-space functions for systems governed by the Hamiltonian (1). For such systems the results of $[25,16]$ can be improved. The averaging over the initial torus is dealt with in section 3 , where we finally give a short conclusion and discussion.

\section{Adiabatic invariance and averaging of phase-space functions}

Suppose that the Hamiltonian (1) is defined for $(J, \varphi) \in G \times \mathbb{T}^{n}$, where $G$ is an open bounded subset of $\mathbb{R}^{n}$. We assume $h_{0}$ and $h_{1}$ to be $\mathscr{C}^{\infty}\left(\bar{G} \times \mathbb{T}^{n} \times[0, T]\right)$, where $\bar{G}$ is the closure of $G$, and by $\mathscr{C}^{\infty}\left(\bar{G} \times \mathbb{T}^{n} \times[0, T]\right)$ we mean that $h_{0}$ and $h_{1}$ have all derivatives on $G \times \mathbb{T}^{n} \times[0, T]$ with bounded extensions to the closure. Assume, moreover, $h_{1}$ to have only finitely many Fourier components, i.e. there exists $K \in \mathbb{Z}^{+}$such that

$$
h_{1}(J, \varphi, \tau)=\sum_{v \in \mathbb{Z}^{n},|v| \leqslant K} h_{1, \nu}(J, \tau) \mathrm{e}^{\mathrm{i} v \cdot \varphi}
$$

where $|v|:=\left|v_{1}\right|+\ldots+\left|v_{n}\right|$ for vectors $v \in \mathbb{Z}^{n}$.

Both these assumptions are not optimal, but simplify our arguments considerably, making them less technical and allowing a clearer presentation of the ideas behind them. As we will remark, one can easily adapt our proof to the case where $h$ is only of class $\mathscr{C}^{m}$ and $h_{1}$ has infinitely many Fourier components.

Let $a(J, \varphi, \tau)$ be a real valued phase-space function of the Hamiltonian system (1) verifying the same assumptions as $h_{1}$. We define

$$
A(t):=\frac{\varepsilon}{T} \int_{0}^{t} a(J(u), \varphi(u), \varepsilon u) \mathrm{d} u \quad \vec{A}(t):=\frac{\varepsilon}{T} \int_{0}^{t} a^{\mathrm{av}}\left(J^{0}, \varepsilon u\right) \mathrm{d} u
$$

where $a^{\mathrm{av}}(J, \tau):=\int_{0}^{2 \pi} \ldots \int_{0}^{2 \pi} a(J, \varphi, \tau) \mathrm{d}^{n} \varphi /(2 \pi)^{n}$ is the average of $a$ over the (fast) angular variables $\varphi \in \mathbb{T}^{n}$. When $t=T / \varepsilon, A(t)$ and $\bar{A}(t)$ denote respectively the time average of $a(I, \varphi, \varepsilon)$ and of its average over the (fast) angular variables during one adiabatic cycle. In the end we will identify $a$ with the components of $g=\nabla_{J} h_{1}$.

By \|\|$_{0}$ we denote the maximum norm. Let $J=\left(J^{(1)}, \ldots, J^{(n)}\right)$. If $b \in \mathscr{C}^{\infty}(\bar{G} \times$ $\left.\mathbb{T}^{n} \times[0, T], \mathbb{R}^{m}\right)$ and $l \in \mathbb{N}_{0}:=\mathbb{N} \cup\{0\}$, we define the seminorm

$$
\|b\|_{l}^{J}:=\max _{|\beta|=l}\left\|\frac{\partial^{l} b}{\partial^{\beta_{1}} J^{(1)} \ldots \partial^{\beta_{n}} J^{(n)}}\right\|_{0}
$$

where clearly the multi-index $\beta \in \mathbb{N}_{0}^{n}$. $\|b\|_{l}^{\varphi}$ is defined analogously, except that the derivatives are taken with respect to $\varphi$. 
With these assumptions and definitions we can prove the adiabatic invariance of the actions $J$ of the Hamiltonian (1) and an averaging theorem for phase-space functions in the spirit of the existence proof for Hannay angles given in $[13,16]$. As we will remark, whereas for the former the non-resonance condition (6) which we will impose can be replaced by the assumption that the Hessian of the unperturbed Hamiltonian $h_{0}$ is convex, thus obtaining a result for all initial conditions, the latter needs the non-resonance condition crucially since it is based on the procedure of elimination of non-resonant harmonics (see, for instance, [3] chapter 5). Moreover, the adiabatic invariance of the actions holds true for time scales much longer than $1 / \varepsilon$ (in fact, possibly exponentially long), whereas the averaging procedure for phase-space functions gives errors of $O(1)$ already for time scales of order $1 / \varepsilon^{2}$. We stress that we obtain a better result for the conservation of the actions than shown in [16] because of our assumption that $h_{0}$ be not explicitly time dependent.

Proposition 1. Consider all initial conditions $\left(J^{0}, \varphi^{0}\right) \in G \times \mathbb{T}^{n}$ such that $J(t) \in G$ for all times $t \in[0, T / \varepsilon]$ and verifies the non-resonance condition

$$
|\omega(J(t)) \cdot v|>\alpha
$$

for all $v \in \mathbb{Z}^{n} \backslash\{0\},|v| \leqslant K$ and for some $\alpha \in(0,1)$. If $\varepsilon$ is sufficiently small, then one has

$$
\begin{aligned}
& \max _{t \in[0, T / \varepsilon]}\left|J(t)-J^{0}\right| \leqslant c_{1} \frac{\varepsilon}{\alpha^{2}} \\
& \max _{t \in[0, T / \varepsilon]}|A(t)-\bar{A}(t)| \leqslant c_{2} \frac{\varepsilon}{\alpha^{2}}
\end{aligned}
$$

where $c_{1}$ and $c_{2}$ are two positive constants independent of $\alpha$ and $\varepsilon$.

We note that our non-resonance assumption depends on the knowledge of the time evolution of $J(t)$, which is clearly unknown in general. However, if $h_{0}$ is non-degenerate or corresponds to a system of harmonic oscillators, one can easily verify (6), as shown in remarks 1 and 2 .

Proof. The proofs use standard techniques of first-order canonical perturbation theory and will only be sketched. These tools are presented in detail in [11,23].

We look for a near-to-identity canonical transformation to new action-angles variables $\left(J^{\prime}, \varphi^{\prime}\right)=\mathscr{C}(J, \varphi ; \tau, \varepsilon)$ with generating function

$$
S\left(J^{\prime}, \varphi ; \tau, \varepsilon\right)=J^{\prime} \cdot \varphi+\varepsilon S_{1}\left(J^{\prime}, \varphi, \tau\right)
$$

such that

$$
h \circ \mathscr{C}^{-1}+\varepsilon^{2} \frac{\partial S_{1}}{\partial \tau}=h_{0}^{\prime}\left(J^{\prime}\right)+\varepsilon h_{1}^{\prime}\left(J^{\prime}, \tau\right)+R_{2}\left(J^{\prime}, \varphi^{\prime}, \tau, \varepsilon\right) .
$$

The generating function (9) gives rise to the canonical transformation

$$
\begin{aligned}
& J=J^{\prime}+\varepsilon \frac{\partial S_{1}}{\partial \varphi}\left(J^{\prime}, \varphi ; \tau\right) \\
& \varphi^{\prime}=\varphi+\varepsilon \frac{\partial S_{1}}{\partial J^{\prime}}\left(J^{\prime}, \varphi ; \tau\right) .
\end{aligned}
$$


If one inserts (11) into (10) one finds that $h_{0}^{\prime}\left(J^{\prime}\right)=h_{0}\left(J^{\prime}\right)$. Moreover, $S_{1}$ has to solve the following homological equation:

$$
\omega\left(J^{\prime}\right) \frac{\partial S_{1}}{\partial \varphi}\left(J^{\prime}, \varphi ; \tau\right)+h_{1}\left(J^{\prime}, \varphi, \tau\right)=h_{1}^{\prime}\left(J^{\prime}, \tau\right) .
$$

The non-resonance condition (6) insures that (13) can be solved:

$$
\begin{aligned}
& h_{1}^{\prime}\left(J^{\prime}, \tau\right)=\int_{\mathbb{T}^{n}} h_{1}\left(J^{\prime}, \varphi, \tau\right) \frac{\mathrm{d}^{n} \varphi}{(2 \pi)^{n}} \\
& S_{1}\left(J^{\prime}, \varphi ; \tau\right)=\sum_{\nu \in \mathbb{Z}^{n} \backslash\{0\},|v| \leqslant K} \frac{h_{1, \nu}\left(J^{\prime}, \tau\right)}{-\mathrm{i} v \omega\left(J^{\prime}\right)} \mathrm{e}^{i v \cdot \varphi}
\end{aligned}
$$

where $h_{1, v}$ denotes the $v$ th Fourier component of $h_{1, v}$, i.e. $h_{1, v}\left(J^{\prime}, \tau\right)=$ $\int_{\mathbb{J}^{n}} h_{1}\left(J^{\prime}, \varphi, \tau\right) \mathrm{e}^{-\mathrm{i} v \cdot \varphi} \mathrm{d}^{n} \varphi /(2 \pi)^{n}$.

Applying the implicit function theorem and choosing $\varepsilon$ sufficiently small, one finds that the canonical transformation $\mathscr{C}(, ; \tau, \varepsilon)$ is well defined, invertible and satisfies

$$
\begin{aligned}
& \left|J-J^{\prime}\right|=\mathscr{O}\left(\frac{\varepsilon}{\alpha}\right) \\
& \left\|R_{2}\right\|_{1}^{\varphi}=\left\|h \circ \mathscr{C}^{-1}+\varepsilon^{2} \frac{\partial S_{1}}{\partial \tau}-h_{0}^{\prime}-\varepsilon h_{1}^{\prime}\right\|_{1}^{\varphi}=\mathscr{O}\left(\frac{\varepsilon^{2}}{\alpha^{2}}\right) .
\end{aligned}
$$

Since, by (10), we have $\mathrm{d} J^{\prime} / \mathrm{d} t=-\partial R_{2} / \partial \varphi$, (7) follows immediately from (14) and (15).

To prove (8) we only need to adapt the argument from [16]. From the definitions of $A(t)$ and $\bar{A}(t)$, one has

$$
\begin{aligned}
A(t)-\bar{A}(t)= & \frac{\varepsilon}{T} \int_{0}^{t}\left[a^{\mathrm{av}}(J(u), \varepsilon u)-a^{\mathrm{av}}\left(J^{0}, \varepsilon u\right)\right] \mathrm{d} u \\
& +\frac{\varepsilon}{T} \int_{0}^{t} \tilde{a}(J(u), \varphi(u), \varepsilon u) \mathrm{d} u
\end{aligned}
$$

where $\tilde{a}(J, \varphi, \varepsilon t):=a(J, \varphi, \varepsilon t)-a^{\text {av }}(J, \varepsilon t)$ denotes the oscillating part of $a$. The first term on the right-hand side of (16) can be immediately bounded uniformly with respect to $t \in[0, T / \varepsilon]$ by means of (7):

$$
\left|\frac{\varepsilon}{T} \int_{0}^{t}\left[a^{\mathrm{av}}(J(u), \varepsilon u)-a^{\mathrm{av}}\left(J^{0}, \varepsilon u\right)\right] \mathrm{d} u\right| \leqslant n c_{1}\left\|a^{\mathrm{av}}\right\|_{1}^{J} \frac{\varepsilon}{\alpha^{2}}
$$

whereas in order to give a bound to the last term we solve, again, a homological equation

$$
\tilde{a}-\omega \nabla_{\varphi} w=0 .
$$

In fact, exploiting again our non-resonance condition (6), it follows immediately that (17) has the solution

$$
w(J, \varphi, \tau)=\sum_{v \in \mathbb{Z}^{n} \backslash\{0\},|v| \leqslant K} \frac{a_{\nu}(J, \tau)}{\mathrm{i} v \cdot \omega(J)} \mathrm{e}^{\mathrm{i} v \cdot \varphi}
$$


where $a_{v}$ denotes the $v$ th Fourier component of $a . w$ satisfies

$$
\begin{aligned}
& \|w\|_{0}=\mathcal{O}\left(\frac{1}{\alpha}\right) \\
& \left\|\nabla_{J} w\right\|_{0}=\mathcal{O}\left(\frac{1}{\alpha^{2}}\right) \\
& \left\|\nabla_{\varphi} w\right\|_{0}=\mathcal{O}\left(\frac{1}{\alpha}\right) \\
& \left\|\frac{\partial w}{\partial \tau}\right\|_{0}=\mathcal{O}\left(\frac{1}{\alpha}\right) .
\end{aligned}
$$

If we now define

$$
\psi:=\frac{\mathrm{d} w}{\mathrm{~d} t}=\omega \cdot \nabla_{\varphi} w+\varepsilon\left(f \nabla_{J} w+g \cdot \nabla_{\varphi} w+\frac{\partial w}{\partial \tau}\right)
$$

then

$$
0=\frac{\varepsilon}{T}\left(w(J(t), \varphi(t), \varepsilon t)-w\left(J^{0}, \varphi^{0}, 0\right)-\int_{0}^{t} \psi(J(u), \varphi(u), \varepsilon u) \mathrm{d} u\right)
$$

and by adding it to the last term on the right-hand side of (16), since $w$ solves the homological equation (17), we obtain

$$
\begin{aligned}
\left\|\frac{\varepsilon}{T} \int_{0}^{t} \tilde{a}(J(u), \varphi(u), \varepsilon u) \mathrm{d} u\left|\leqslant \frac{2 \varepsilon}{T}\|w\|_{0}+\frac{\varepsilon}{T}\right| \int_{0}^{t}(\tilde{a}-\psi)(J(u), \varphi(u), \varepsilon u) \mathrm{d} u \mid\right. \\
\quad \leqslant \frac{2 \varepsilon}{T}\|w\|_{0}+\varepsilon n\left(\|f\|_{0}\left\|\nabla_{J} w\right\|_{0}+\|g\|_{0}\left\|\nabla_{\varphi} w\right\|_{0}\right)+\varepsilon\left\|\frac{\partial w}{\partial \tau}\right\|_{0} \\
=\mathcal{O}\left(\frac{\varepsilon}{\alpha^{2}}\right) .
\end{aligned}
$$

Let us compare our proof with the one given in [16] (or the extension in $[4,9]$, where the case of non-independent frequencies is considered), which followed Neishtadt's original approach [25]. One realises that the assumption that $h_{0}$ be not explicitly time dependent allows for introducing the non-resonance condition (6) since manifolds of constant action are either resonant or non-resonant. This gives the possibility of a pointwise solution of the homological equations (13), (17) and leads to a better $\varepsilon$ dependence for estimates (7) and (8).

Remark 1. Non-degenerate $h_{0}$. If $h_{0}$ is non-degenerate, i.e. if there exists a positive constant $d<\infty$ such that for all $J \in G$

$$
\left|\operatorname{det}\left(\frac{\partial^{2} h_{0}}{\partial J^{(i)} \partial J^{(k)}}\right)(J)\right| \geqslant d^{-1}>0
$$

as is well known, the complement of the non-resonant set

$$
\hat{G}:=\left\{J \in G|| \omega v \mid>\alpha \text { for all } v \in \mathbb{Z}^{n} \backslash\{0\},|v| \leqslant K\right\}
$$

has a Lebesgue measure

$$
\operatorname{meas}(G \backslash \hat{G}) \leqslant c_{3} d K^{n} \alpha
$$


for some $c_{3}>0$. For these systems one only needs to verify a non-resonance condition at time $t=0$ : imposing that

$$
\left|\omega\left(J^{0}\right) \cdot v\right|>2 \alpha
$$

for all $|v| \leqslant K, v \neq 0$, one can easily check that (6), and therefore (7) and (8), still hold true, provided that $\varepsilon$ is sufficiently small.

Thus, from the estimate on the measure of the non-resonant set (22), one finds that (7) and (8) are valid for all initial conditions outside a set of Lebesgue measure of order $\alpha$. Since the constants are independent of $\varepsilon$ and $\alpha$, one can also choose $\alpha=\varepsilon^{a}$ for any $a \in\left[0, \frac{1}{2}\right)$ thus obtaining that the actions are almost adiabatic invariants for all initial conditions outside a set whose measure vanishes in the limit $\varepsilon \rightarrow 0$, and that the geometrical Hannay angles (4) are given by

$$
\lim _{\varepsilon \rightarrow 0}\left[\varphi\left(\frac{T}{\varepsilon}\right)-\varphi^{0}-\int_{0}^{T / \varepsilon} \omega(J(t)) \mathrm{d} t\right]
$$

for almost every initial condition $\left(J^{0}, \varphi^{0}\right) \in G \times \mathbb{T}^{n}$.

Remark 2. Harmonic oscillators. If the original unperturbed Hamiltonian describes a system of harmonic oscillators, i.e.

$$
h_{0}(J)=\omega \cdot J
$$

then the frequency vector $\omega \in \mathbb{R}^{n}$ is independent of $J$. In this case the non-resonance condition (6) is just a condition on $\omega \in \mathbb{R}^{n}$ and is automatically verified for all times. Therefore (7) and (8) hold whenever

$$
|\omega \cdot v| \geqslant \alpha
$$

for all $v \in \mathbb{Z}^{n} \backslash\{0\},|\beta| \leqslant K$. Moreover, if $\omega$ is highly non-resonant so that it verifies a Diophantine condition, i.e. there exist $\gamma>0$ and $\mu \geqslant n-1$ such that

$$
|\omega \cdot v| \geqslant \gamma|v|^{-\mu}
$$

for all $v \in \mathbb{Z}^{n} \backslash\{0\}$, one can prove that the actions are adiabatic invariants for exponentially long times by adapting an argument due to Gallavotti [11] (see also $[5,6,12])$, provided that $h$ has an analytic extension to a complex neighbourhood of $G \times \mathbb{T}^{n} \times[0, T]$ (maintaining periodicity in $\tau$ ). The key idea is to construct the non-resonant normal form for the Hamiltonian $h$, as shown in remark 4 , and to choose an optimal truncation order of the power series expansion (24) in $\varepsilon$ in a way such that the norm of the remainder is minimised.

We also refer to the work of Leung and Meyer [22] and Fedoryuk [10] who proved the adiabatic invariance of the actions to all orders (or even exponential adiabatic invariance [10]) for the case of harmonic oscillators with slowly timedependent frequencies.

Remark 3. Time scales for averaging of phase-space functions. Let us consider the simple one-degree-of-freedom system with Hamiltonian $h(J, \varphi)=J+\varepsilon \cos \varphi$. The equations of the motion for any initial condition $\left(J^{0}, \varphi^{0}\right)$ are trivially integrated and give $J(t)=J^{0}-\varepsilon \cos \left(\varphi^{0}+t\right)+\varepsilon \cos \varphi^{0}, \varphi(t)=\varphi^{0}+t$. Clearly $\left|J(t)-J^{0}\right|=\mathcal{O}(\varepsilon)$ for all times, but if one chooses the phase-space function $a=J^{2}$, one finds

$$
A(t)-\bar{A}(t)=\frac{\varepsilon}{T} \int_{0}^{t}\left[J^{0}+\varepsilon \cos \varphi^{0}-\varepsilon \cos \left(\varphi^{0}+t\right)\right]^{2} \mathrm{~d} t-\frac{\varepsilon\left(J^{0}\right)^{2} t}{T}
$$

which is $\mathcal{O}(1)$ already at $t=1 / \varepsilon^{2}$. 
This example shows that whilst (7) may hold true for much longer time scales, estimate (8) is in general wrong for $t>1 / \varepsilon$.

Remark 4. Time scales for adiabatic invariance of actions. The example given in the previous remark suggests that (7) might hold true on longer time scales. In fact, if the non-resonance condition (6) is verified by $J(t)$ for all $t$ with $0 \leqslant t \leqslant \varepsilon^{-r}$ for some $r \in \mathbb{N}_{0}$ one can prove that (7) holds for the same time scale by higher-order canonical perturbation theory.

Again, the idea is to find a near-to-identity canonical transformation to new action-angle variables $\left(J^{\prime}, \varphi^{\prime}\right)=\mathscr{C}(J, \varphi ; \tau, \varepsilon)$ generated by

$$
S\left(J^{\prime}, \varphi, \tau, \varepsilon\right)=J^{\prime} \cdot \varphi+\sum_{l=1}^{r} \varepsilon^{l} S_{l}\left(J^{\prime}, \varphi, \tau\right)
$$

for some $r \geqslant 1$, such that

$$
\left(h \circ \mathscr{C}^{-1}\right)+\varepsilon^{2} \frac{\partial S}{\partial \tau}=h_{0}^{\prime}\left(J^{\prime}\right)+\sum_{l=1}^{r} \varepsilon^{l} h_{l}^{\prime}\left(J^{\prime}, \tau\right)+R_{r+1}\left(J^{\prime}, \varphi^{\prime}, \tau, \varepsilon\right) .
$$

A power expansion in $\varepsilon$ shows that at all orders $\varepsilon^{l}$ with $1 \leqslant l \leqslant r$ one must solve

$$
\omega\left(J^{\prime}\right) \cdot \nabla_{\varphi} S_{l}\left(J^{\prime}, \varphi, \tau\right)+Q_{l}\left(J^{\prime}, \varphi, \tau\right)=h_{l}^{\prime}\left(J^{\prime}, \tau\right)
$$

where $Q_{l}$ depends only on $S_{1}, \ldots, S_{l-1}$. Clearly the non-resonance condition ensures that (25) can be solved and the remainder $R_{r+1}$ is determined by means of the inverse function theorem

$$
R_{r+1}:=h \circ \mathscr{C}^{-1}+\varepsilon^{2} \frac{\partial S}{\partial \tau}-\sum_{l=0}^{r} \varepsilon^{l} h_{l}^{\prime}
$$

One can verify that

$$
\left|J^{\prime}-J\right|=\mathcal{O}\left(\frac{\varepsilon}{\alpha}\right) \quad\left\|R_{r+1}\right\|_{0}=\mathcal{O}\left(\frac{\varepsilon^{r+1}}{\alpha^{m}}\right)
$$

for some $m \geqslant r$, and one finds that (7) holds true, indeed, for all times $t \in\left[0,(T / \varepsilon)^{r}\right]$. We stress that in order to develop this standard perturbative scheme the assumption that $h_{0}$ is $\tau$ independent is essential.

Remark 5. Non-degenerate $h_{0}$ : adiabatic invariance of actions for exponentially long time scales. For non-degenerate Hamiltonian systems with one degree of freedom undergoing an adiabatic parameter change, Arnol'd showed [1] that KAM theory ensures that the action is a perpetual adiabatic invariant, i.e. it is invariant for all times. When the number of degrees of freedom exceeds one, examples show [3] that, in general, for a set of initial conditions whose Lebesgue measure is of order 1 the actions can undergo a variation of order 1 over a time scale of $\varepsilon^{-3 / 2}$. The slow variation of the parameters causes the system to very often experience structural transitions: a constant non-resonant action may become resonant and vice versa due to the explicit time dependence of $h_{0}$. The resonant tori are generally destructed by the adiabatic change and give an everywhere dense set of bifurcation regions in phase space. However, if $h_{0}$ does not depend explicitly on $\tau$, as for (1), this cannot occur and, in fact, if $h$ has an analytic extension to a complex neighbourhood of $G \times \pi \times[0, T]$ (maintaining periodicity in $\tau$ ) one can show by means of phase-space 
perturbation theory that whenever $\omega\left(J^{0}\right)$ is non-resonant, i.e. verifies (23), one has

$$
\left|J(t)-J^{0}\right| \leqslant c_{4} \varepsilon^{c_{5}}
$$

for $0 \leqslant t \leqslant c_{6} \exp \left\{\left(\varepsilon^{*} / \varepsilon\right)^{c_{7}}\right\}$, where $c_{4}, c_{5}, c_{6}$ and $c_{7}$ are positive constants.

Moreover, if one assumes that $h_{0}$ is not only non-degenerate but also convex (or, more generally, verifies a steepness condition [26]), i.e. there exists $d^{\prime}>0$ such that for all $J \in G$ and for all $v \in \mathbb{R}^{n}$,

$$
\sum_{i, k=1}^{n} \frac{\partial^{2} h_{0}}{\partial J^{(i)} \partial J^{(k)}}(J) v^{(i)} v^{(k)} \geqslant d^{\prime} \sum_{i=1}^{n}\left(v^{(i)}\right)^{2}
$$

the non-resonance condition can be dropped, as remarked by Nekhoroshev [26]: (26) holds true for all initial conditions and for exponentially long times for non-autonomous systems for which the perturbation depends on the slow time $\varepsilon$, i.e. for systems with the Hamiltonian (1).

The readers familiar with the proof of the Nekhoroshev theorem in the autonomous case will find the proof of this statement absolutely evident. For a proof of the Nekhoroshev theorem in the autonomous case we refer to $[6,11,26]$ for Hamiltonian flows and to [5] for symplectic maps. It should be noted, however, that two assumptions are absolutely essential in addition to the convexity of $h_{0}$. First, $h$ must be analytic, since if we only require it to be smooth then the estimate is not exponential but a power estimate (as in remark 2). Second, for the non-autonomous system we are interested in, the time independence of $h_{0}$ plays a crucial rôle.

To conclude, we stress that in contrast to the adiabatic invariance of the actions, the averaging theorem (8) for phase-space functions needs a non-resonance condition, since we want to average over the full $n$-dimensional torus. If the system is locked in some resonance one might, however, apply partial averaging on some embedded $\mathbb{T}^{n-m}$ torus, where $m$ is the dimension of the resonant module.

Remark 6. Optimality of the assumptions. The assumption that $h_{1}$ has only finitely many Fourier components can be made without loss of generality as we assumed $h_{1}$ to be smooth: in fact, one might allow $h_{1}$ to have infinitely many Fourier components and introduce an ultraviolet cut-off $K(\varepsilon)$. Split the Fourier series into two parts $h_{1}^{\leqslant}$and $h_{1}^{>}$which contain, respectively, modes satisfying $|v| \leqslant K(\varepsilon)$ and $|v|>K(\varepsilon)$. Then one finds that $\left\|h_{1}^{>}\right\|_{0}=\mathcal{O}(\varepsilon)$, provided $K(\varepsilon)=\mathcal{O}\left(\varepsilon^{-\delta}\right)$, for any $\delta>0$, because the norm of the Fourier modes decays faster than any power of $|v|$, Therefore $\left\|\varepsilon h_{1}^{>}\right\|_{0}=\mathcal{O}\left(\varepsilon^{2}\right)$ would not affect the dynamics sensibly for the time scale $0 \leqslant t \leqslant T / \varepsilon$ of interest.

Moreover, one can drop the $\mathscr{C}^{\infty}$ assumptions. It suffices to assume $h_{1} \in \mathscr{C}^{m}(\tilde{G} \times$ $\left.\mathbb{T}^{n} \times[0, T]\right)$ and $a \in \mathscr{C}^{m-1}\left(\bar{G} \times \mathbb{T}^{n} \times[0, T]\right)$ with $m \geqslant n+3$ and one may allow $h_{1}$ to have infinitely many Fourier components. In this case one has to choose

$$
K(\varepsilon)=O\left(\varepsilon^{-1 /(m-n-1)}\right)
$$

and obtain the statement of theorem 1 . If one considers non-degenerate $h_{0}$, as in remark 1 , one needs to make sure that the measure of the non-resonant set $\hat{G}$ vanishes for $\varepsilon \rightarrow 0$ (and $\alpha=\varepsilon^{a}$ ). This makes it necessary to require $m \geqslant 3 n+1$. In fact, one needs

$$
\frac{n}{m-n-1}<a<\frac{1}{2}
$$




\section{Averaging over initial angles}

The goal of this section is to prove an averaging theorem; averaging refers in this context to the initial angles $\varphi^{0}$. For the class of systems we are considering this will enable us to obtain an a priori estimate for the averaged dynamical angles $\left\langle\int_{0}^{t} \omega(J(u), \varepsilon u) \mathrm{d} u\right\rangle$. By $\langle\cdot\rangle$ we have denoted the average over the initial angles $\varphi^{0}$.

Theorem 1. Let the same assumptions as in proposition 1 be satisfied and let $\Omega(J, \tau)$ be in $\mathscr{C}^{\infty}(\bar{G} \times[0, T])$. Then there exists a constant $c_{8}$ such that

$$
\max _{t \in[0, T / \varepsilon]}\left|\left\langle\int_{0}^{t} \Omega(J(u), \varepsilon u) \mathrm{d} u\right\rangle-\int_{0}^{t} \Omega\left(J^{0}, \varepsilon u\right) \mathrm{d} u\right| \leqslant c_{8} \frac{\varepsilon}{\alpha^{4}} .
$$

Proof. Let $\left(J^{0}, \varphi^{0}\right)$ satisfy the assumptions of proposition 1 , and set

$$
\Delta:=\int_{0}^{t}\left[\Omega(J(u), \varepsilon u)-\Omega\left(J^{0}, \varepsilon u\right)\right] \mathrm{d} u .
$$

Upon a Taylor expansion of $\Omega(J, \tau)$ in $J$ and applying the adiabatic theorem (formula (7)) one obtains, always assuming that $t \in[0, T / \varepsilon]$,

$$
\Delta=\int_{0}^{t}\left(J(u)-J^{0}\right) \cdot \nabla J \Omega\left(J^{0}, \varepsilon u\right) \mathrm{d} u+\mathcal{O}\left(\frac{\varepsilon}{\alpha^{4}}\right) .
$$

Here and in the following $\mathcal{O}(\cdot), \varepsilon \rightarrow 0$, is always meant uniformly in $t \in[0, T / \varepsilon]$ and $\left(J^{0}, \varphi^{0}\right)$.

Because of the non-resonance assumption (6) second-order canonical perturbation theory can be used to prove the existence of a near-to-identity canonical transformation to new action-angle coordinates $\left(J^{\prime}, \varphi^{\prime}\right)$ verifying

$$
\left|J-J^{\prime}\right|=\mathcal{O}\left(\frac{\varepsilon}{\alpha}\right) \quad \frac{\mathrm{d}}{\mathrm{d} t} J^{\prime}=\mathscr{O}\left(\frac{\varepsilon^{3}}{\alpha^{4}}\right) .
$$

If $J^{\prime} \cdot \varphi+\varepsilon S\left(J^{\prime}, \varphi ; \tau, \varepsilon\right)$ is its generating function, one has also the estimate $\|S\|_{i}^{\varphi}=\mathcal{O}(1 / \alpha)$; thus

$J(u)-J^{0}=\left[J^{\prime}(u)-\left(J^{\prime}\right)^{0}\right]+\varepsilon \frac{\partial S}{\partial \varphi}\left(J^{\prime}(u), \varphi(u) ; \varepsilon u, \varepsilon\right)+\varepsilon \frac{\partial S}{\partial \varphi}\left(\left(J^{\prime}\right)^{0}, \varphi^{0} ; 0, \varepsilon\right)$.

Because of (28) the first term on the RHS is of order $\mathscr{O}\left(\varepsilon^{2} / \alpha^{4}\right)$. For the second contribution we may apply the proposition in section 2 , yielding $\mathcal{O}\left(\varepsilon^{2} / \alpha^{3}\right)$. Finally, the last term vanishes upon averaging over $\varphi^{0}$.

If we now replace in the proposition of theorem 1 the function $\Omega$ by the components of the angular frequencies $\omega$, we get as a corollary that the average over the torus of the dynamical angles is equal to $T \omega\left(J^{0}\right) / \varepsilon$, up to an error of order $\mathcal{O}\left(\varepsilon / \alpha^{4}\right)$.

We have applied an averaging procedure to a class of systems of several degrees of freedom governed by a Hamiltonian $h=h_{0}(J)+\varepsilon h_{1}(J, \varphi, \tau)$. What is the scope of this method?

It seems to us that such an averaging procedure is acceptable from the experimental point of view provided the experimentalist can prepare the system with 
$J^{0}$ fixed but varying $\varphi^{0}$. This premise, however, restricts the experimental applicability of the proposed averaging over the initial torus and limits the extent to which our result has an impact on experimental measurements of Hannay angles. One such a situation where the idea of averaging over initial angles seems not to be applicable is given, for instance, by the motion of celestial bodies, since in celestial mechanics one cannot choose initial conditions freely.

One may also wonder whether a statement in the spirit of our theorem 1 may hold true for general $h_{0}$, i.e. for $h_{0}=h_{0}(J, \tau)$. We do not believe that our result on averaging over the initial torus would continue to hold under these general circumstances. In this situation, as we stressed several times, to a fixed value of $J^{0}$ there corresponds a frequency vector $\omega\left(J^{0}, \tau\right)$ whose resonant or non-resonant character depends on the current value of $\tau$. This makes averaging over the initial torus a questionable procedure. It seems to us that for such general systems it is rather doubtful whether there is any experimental method for determining the anholonomy effect discovered by Hannay.

Our expectation that theorem 1 may not be always true is supported by a Taylor expansion of $\omega(J, \tau)$ up to second order with respect to $J$. Assume that one could treat the first-order term by averaging over initial angles. Note that in this paper the second-order term was not doing any harm because of the better conservation of the actions here (formula (7)): it involves the square of the deviation of the action from the initial action and therefore gives at most a contribution of order $\mathcal{O}\left(\varepsilon / \alpha^{4}\right)$ to the dynamical angles, even without averaging over the initial torus. In general, however, the second-order term is of the order $\mathcal{O}\left(\varepsilon^{2 b}\right)$, where $b \in\left[0, \frac{1}{2}\right)$ (cf [16]), and it is not plausible that averaging over $\varphi^{0}$ would improve the rate of convergence to zero, due to positivity of the square. So it seems that this gives rises to a term of order $\mathcal{O}\left(\varepsilon^{2 b-1}\right)$ in the approximation to the dynamical angles. Since $b<\frac{1}{2}$, this term exceeds the Hannay angles. In our opinion this indicates that theorem 1 may not be true for the case of $h_{0}=h_{0}(J, \tau)$. However, we have not yet an explicit analytical counterexample.

Finally we would like to point out that the problems in the measurement of the Hannay angles do not occur for the Berry phase. This is discussed in [14].

\section{Acknowledgements}

One of us (SG) would like to thank his colleagues in Bologna (Dipartimento di Fisica and Istituto di Fisica Nucleare, Università di Bologna) for their hospitality. Thanks go also to Andreas Knauf for many discussions and to Giorgio Mantica for a critical reading of the manuscript. We also like to thank an unknown referee for a suggestion how to simplify the proof of theorem 1 considerably. On the part of SG this work has been supported by the Deutsche Forschungsgemeinschaft.

\section{References}

[1] Arnol'd V I 1963 Small denominators and problems of stability of motion in classical and celestial mechanics Usp. Mat. Nauk 18 91-192 (Engl. transl. 1963 Russ. Math. Surv. 18 85-191)

[2] Arnol'd V I 1983 Geometrical Methods in the Theory of Ordinary Differential Equations (Berlin: Springer) 
[3] Arnol'd V I, Kozlov V V and Neishtadt A I 1988 Mathematical Aspects of Classical and Celestial Mechanics, Encyclopaedia of Mathematical Sciences, vol 3: Dynamical Systems, III (Berlin: Springer)

[4] Bakhtin V I 1985 Averaging in multifrequency systems Funkts. Anal. Prilozh. 20 1-7 (Engl. transl. 1986 Func. Anal. Appl. 20 83-8)

[5] Bazzani A, Marmi S and Turchetti G 1989 Nekhoroshev estimate for isochronous non-resonant symplectic maps Preprint University of Bologna

[6] Benettin G and Gallavotti G 1986 Exponential estimate for the stability times in nearly-integrable Hamiltonian systems $J$. Stat. Phys. 44 293-338

[7] Berry M V 1984 Quantal phase factors accompanying adiabatic changes Proc. R. Soc. A 392 45-57

[8] Berry M V 1985 Classical adiabatic angles and quantal adiabatic phase J. Phys. A: Math. Gen. 18 $15-27$

[9] Dodson M M, Rynne B P and Vickers J A G 1989 Averaging in multifrequency systems Nonlinearity 2 137-48

[10] Fedoryuk M V 1976 An adiabatic invariant of a system of linear oscillators and scattering theory Differ. Uraun. 12 1012-8 (Engl. transl. 1976 Differ. Equations 12 713-8)

[11] Gallavotti G 1986 Quasi-integrable mechanical systems Phénomènes critiques, systèmes aléatoires, théories de jauge ed K Osterwalder and R Stora (Proc. Les Houches Summer School, Session XLIII) (Amsterdam: Elsevier)

[12] Giorgilli A and Galgani L 1985 Rigorous estimates for the series expansions of Hamiltonian perturbation theory Cel. Mech. 37 95-112

[13] Golin S 1988 Existence of the Hannay angle for single-frequency systems J. Phys. A: Math. Gen. 21 4535-47

[14] Golin S 1989 Can one measure Hannay angles? J. Phys. A: Math. Gen. 22 4573-80

[15] Golin S, Knauf A and Marmi S 1989 Hannay angles: existence and geometrical interpretation Conference of Nonlinear Dynamics ed G Turchetti (Singapore: World Scientific) pp 200-9

[16] Golin S, Knauf A and Marmi S 1989 The Hannay angles: geometry, adiabaticity, and an example Commun. Math. Phys. 123 95-122

[17] Golin S, Knauf A and Marmi S 1990 Hannay angles and classical perturbation theory Number Theory and Physics ed J M Luck, P Moussa and M Waldschmidt (Lectures Notes in Physics) (Berlin: Springer)

[18] Golin S and Marmi S 1989 Symmetries, Hannay angles and precession of orbits Europhys. Lett. 8 399-404

[19] Hannay J H 1985 Angle variable holonomy in adiabatic excursion of an integrable Hamitonian J. Phys. A: Math. Gen. 18 221-30

[20] Kugler M 1987 Motion in non-inertial systems, theory and demonstrations Preprint Weizmann Institute of Science, Rehovot)

[21] Kugler M and Shtrikman S 1988 Berry's phase, locally inertial frames, and classical analogues Phys. Rev. D 37 934-7

[22] Leung A and Meyer K 1975 Adiabatic invariants for linear Hamiltonian systems J. Diff. Eqns. 17 $32-43$

[23] Lochak P and Meunier C 1988 Multiphase Averaging for Classical Systems; With Applications to Adiabatic Theorems (Berlin: Springer)

[24] Montgomery R 1988 The connection whose holonomy is the classical adiabatic angles of Hannay and Berry and its generalization to the non-integrable case Commun. Math. Phys. 120 269-94

[25] Neishtadt A I 1976 Averaging in multifrequency systems. II. Dokl. Akad. Nauk. SSSR 226 1295-8 (Engl. transl. 1976 Sov. Phys. Dokl. 21 80-2)

[26] Nekhoroshev N N 1977 Exponential estimate of the stability time of near-integrable Hamiltonian systems Russ. Math. Surv. 32 1-65

[27] Weinstein A 1988 Connections of Berry and Hannay type for moving Lagrangian manifolds Preprint Berkeley 